\title{
Hydromagnetic stagnation point flow over a porous stretching surface in the presence of radiation and viscous dissipation
}

\author{
Emmanuel Maurice Arthur ${ }^{*}$, Ibrahim Yakubu Seini \\ Mathematics Department, Faculty of Mathematical Sciences, Navrongo Campus, Ghana \\ Email address: \\ jimknights01@yahoo.com (E. M. Arthur), yakubuseini@yahoo.com (I. Y. Seini)
}

\section{To cite this article:}

Emmanuel Maurice Arthur, Ibrahim Yakubu Seini. Hydromagnetic Stagnation Point Flow over a Porous Stretching Surface in the Presence of Radiation and Viscous Dissipation. "pplied and Computational Mathematics. Vol. 3, No. 5, 2014, pp. 191-196.

doi: $10.11648 /$ j.acm.20140305.11

\begin{abstract}
This paper investigates the hydromagnetic stagnation point flow of an incompressible viscous electrically conducting fluid towards a stretching sheet in the presence of radiation and viscous dissipation. The Newton-Raphson shooting method along with the fourth-order Runge-Kutta integration algorithm has been employed to tackle the third order, nonlinear boundary layer problem governing the flow. Numerical results for dimensionless local skin friction coefficient and the local Nusselt numbers are presented in tables while graphical results are presented for velocity and temperature profiles for various values of the controlling parameters. The results show that the heat transfer of a hydromagnetic fluid over a porous stretching surface subject to radiation and viscous dissipation can be controlled and a final product with desired characteristics can be achieved.
\end{abstract}

Keywords: Velocity Ratio, Viscous Flow, Radiation, Hydromagnetic, Stagnation Point, Suction

\section{Introduction}

The problem of heat and mass transfer in a laminar boundary layer flow over a stretching sheet in a saturated porous medium finds important application in metallurgy and chemical engineering industries.MHD stagnation point flow of a stretching surface has attracted considerable attention in recent times as it is applied extensively inMHD generators and cooling of infinite metallic plates in cooling baths.

The two-dimensional stagnation point flow problem was first studied by Hiemenz [8] who used the similarity transformations approach to reduce the Navier-Stokes equations to non-linear ordinary differential equations. Ramachandran et al. [18] later investigated the laminar mixed convection in two dimensional stagnation flows around heated surfaces by considering both an arbitrary wall temperature and a varying surface heat flux. Their work was then extended by Devi et al. [5] to the unsteady case and by Lok et al. [15] to a vertical surface immersed in a micropolar fluid. Layek et al. [14] analyzed the flow and heat transfer boundary layer stagnation-point flow of an incompressible and viscous fluid towards a heated porous stretching sheet embedded in porous media subject to suction/blowing with internal heat generation or absorption. Recently, Adrian [1] studied heat and mass transfer by natural convection at a stagnation-point in a porous medium by considering Soret and Dufour effects. Kechil and Hashim [13] earlier studied the boundary layer flow over a nonlinearly stretching sheet in a magnetic field with chemical reaction. Fang et al. [24] then presented analytical results by investigating the hydrodynamic boundary layer flow of slip MHD viscous flow over a stretching sheet and concluded that the wall drag force increases with the magnetic parameter. Ishak et al. [12] studied numerically a steady two-dimensional MHD stagnation point flow towards a stretching sheet with variable surface temperature. They found that the heat transfer rate at the surface increased with the magnetic parameter when the free stream velocity exceeded the stretching velocity. Ibrahim and Makinde [11] investigated the effects of radiation on chemically reacting MHD boundary layer flow past a porous vertical flat plate.

Makinde and Charles [16] then conducted a computational dynamics on the hydrodynamic stagnation point flow towards a stretching sheet and concluded that the cooling rate of a stretching sheet in an electrically conducting fluid, subject to a magnetic field could be controlled and a final product with desired characteristics could be achieved. Ibrahim and 
Makinde [10] also investigated the MHD boundary layer flow of chemically reacting fluid with heat and mass transfer past a stretching sheet and concluded that both the magnetic field strength and the uniform heat source had significant impact on the rate of heat and mass transfer in the boundary layer region. Seini [20] recently investigated the flow over an unsteady stretching surface with chemical reaction and non-uniform heat source. Alireza et al. [2] also presented an analytical solution for MHD stagnation point flow and heat transfer over a permeable stretching sheet with chemical reaction. Recently, Arthur and Seini [3] analyzed the MHD thermal stagnation point flow towards a stretching porous surface. Seini and Makinde [22] then analyzed the boundary layer flow near stagnation-points on a vertical surface with slip in the presence of transverse magnetic field.

Convective heat transfer plays a very important role in practice but in occasions where processes occur at high temperatures, radiation heat transfer cannot be neglected. Hossain and Takhar [9] studied the effect of radiation using the Rosseland diffusion approximation, which leads to non-similar solutions for the forced and free convection flow of an optically dense fluid from vertical surfaces with constant free stream velocity and surface temperature. Pop et al. [17] analyzed the radiative effects on the steady two-dimensional stagnation-point flow of an incompressible fluid over a stretching sheet. Bhattacharyya and Layek [4] investigated the effects of suction/blowing on steady boundary layer stagnation point flow and heat transfer towards a shrinking sheet with thermal radiation. In the same way, the influence of thermal radiation on MHD stagnation point flow past a stretching sheet with heat generation was studied by Zhu et al. [23]. Seini and Makinde [21] later studied the MHD boundary layer flow due to exponential stretching surface with radiation and chemical reaction. Recently, Etwire et al. [7] investigated the MHD boundary layer stagnation point flow with radiation and chemical reaction towards a heated shrinking porous surface.Viscous dissipation plays an important role in geological processes, polymer processing and in strong gravitational field processes on large scales and has received remarkable attention from researchers in fluid mechanics. It plays a practical role in oil products transportation through ducts. Viscous dissipation changes the temperature distribution by acting as an energy source, which affects the heat transfer rate.

The combined effect of radiation and viscous dissipation on stagnation point flow towards a stretching sheet is useful from the practical point of view. This paper investigates the hydromagnetic stagnation point flow over a porous stretching sheet when radiation and viscous dissipation effects are present. Section 2 presents the mathematical model of the problem. The numerical procedure is outlined in section 3 whilst results and discussions are presented in section 4 . Section 5 presents some useful conclusions.

\section{Mathematical Model}

Consider a steady two-dimensional flow of an incompressible and electrically conducting fluid towards the stagnation point on a porous stretching surface in the presence of magnetic field of strength, $B_{o}$, applied in the positive $y$ direction as shown in Figure 1. The tangential velocity $U_{w}$ and the free stream velocity $U_{\infty}$ are assumed to vary proportional to the distance $x$, from the stagnation point so that $U_{w}(x)=b x$ and $U_{\infty}(x)=a x$, where $a$ and $b$ are constants. The induced magnetic field due to the motion of the electrically conducting fluid and the pressure gradient are neglected. The wall temperature is maintained at the prescribed constant value $T_{w}$.

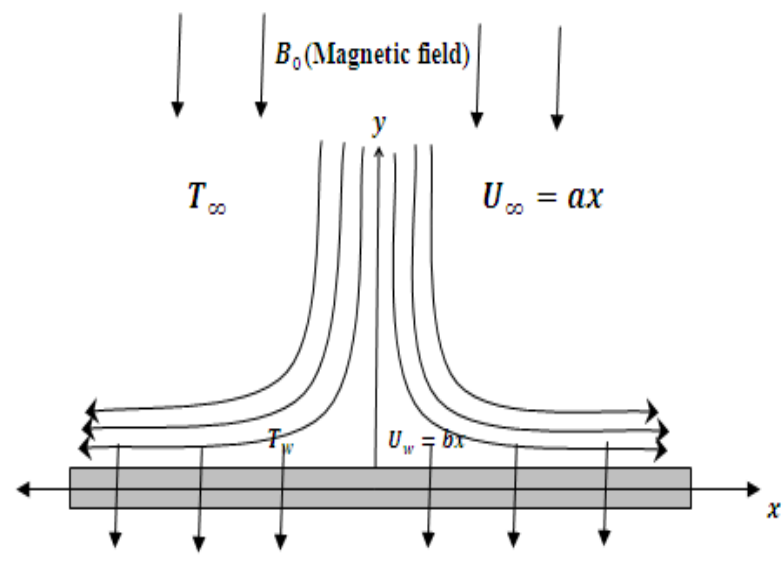

Figure 1. Schematic diagram of the problem.

Under the usual boundary layer approximations, the equations governing the problem of steady, incompressible and viscous flow are:

$$
\begin{gathered}
\frac{\partial \mathrm{u}}{\partial \mathrm{x}}+\frac{\partial \mathrm{v}}{\partial \mathrm{y}}=0 \\
u \frac{\partial u}{\partial x}+v \frac{\partial u}{\partial x}=v \frac{\partial^{2} u}{\partial x^{2}}-\frac{\sigma B_{\circ}^{2}}{\rho} u+a^{2} x, \\
u \frac{\partial T}{\partial x}+v \frac{\partial T}{\partial y}=\alpha \frac{\partial^{2} T}{\partial y^{2}}+\frac{v}{c_{p}}\left(\frac{\partial u}{\partial y}\right)^{2}-\frac{\alpha}{\kappa} \frac{\partial q_{r}}{\partial y}+\frac{\sigma B_{\circ}^{2}}{\rho c_{p}} u^{2} .
\end{gathered}
$$

Boundary conditions:

$$
\begin{gathered}
\mathrm{u}(\mathrm{x}, 0)=\mathrm{bx}, \mathrm{v}(\mathrm{x}, 0)=-\mathrm{v}, T(x, 0)=T_{w}, \\
u(x, \infty)=a x, T(x, \infty)=T_{\infty},
\end{gathered}
$$

where $v$ is the kinematic viscosity, $\sigma$ is the electrical conductivity, $\alpha$ is the thermal diffusivity, $\kappa$ is the thermal conductivity, $\rho$ is the fluid density, $c_{p}$ is the specific heat capacity at constant pressure and $q_{r}$ is the radiative heat flux.

\section{Numerical Procedure}

The velocity components $u$ and $v$ are expressed in terms of the stream function $\psi(x, y)$ in the usual way such that: 


$$
\mathrm{u}=\frac{\partial \psi}{\partial \mathrm{y}}, \quad \mathrm{v}=-\frac{\partial \psi}{\partial \mathrm{x}}
$$

Using the similarity variables:

$$
\psi(x, y)=x \sqrt{v b} f(\eta) \text { and } \eta=y \sqrt{\frac{b}{v}} .
$$

The continuity equation (1) is satisfied identically. Using the Rosseland approximation for radiation, Ibrahim and Makinde [11] simplified the radiation heat flux as

$$
q_{r}=-\frac{4 \sigma^{*}}{3 K^{\prime}} \frac{\partial T^{4}}{\partial y}
$$

where $\sigma^{*}$ and $K^{\prime}$ are the Stefan-Boltzmann constant and the mean absorption coefficient respectively. We assume that the temperature differences within the flow such as the term $T^{4}$ may be expressed as a linear function of temperature. Hence, expanding $T^{A}$ in a Taylor series about $T_{\infty}$ and neglecting higher order terms, we get;

$$
T^{4} \cong 4 T_{\infty}^{3} T-3 T_{\infty}^{4} .
$$

The non-dimensional temperature is written as:

$$
\theta(\eta)=\frac{T-T_{\infty}}{T_{S}-T_{\infty}}
$$

Equations (5) - (9) transform (1) - (4) into the following ordinary nonlinear system of differential equations:

$$
\begin{gathered}
f^{\prime \prime \prime}+f f^{\prime}-\left(M+f^{\prime}\right) f^{\prime}=-\lambda^{2}, \\
\left(1+\frac{3}{4} R a\right) \theta^{\prime \prime}+\operatorname{Pr} f \theta^{\prime}+B r\left(M f^{\prime 2}+f^{\prime \prime 2}\right)=0 .
\end{gathered}
$$

The associated boundary conditions then become:

$$
\begin{gathered}
f^{\prime}(0)=1, \quad f(0)=f_{w}, \theta(0)=1, \\
f^{\prime}(\infty)=\lambda, \theta(\infty)=0,
\end{gathered}
$$

where primes denote the order of differentiation with respect to $\eta, \lambda=\frac{\mathrm{a}}{\mathrm{b}}$ is the velocity ratio parameter, $\operatorname{Pr}=\frac{v}{\alpha}$ is the Prandtl number, $R a=\frac{4 \sigma^{*} T_{\infty}^{3}}{\kappa K^{\prime}}$ is the thermal radiation parameter, $M=\frac{\sigma B_{0}^{2}}{\rho b}$ is the magnetic field parameter, $f_{w}=-v / \sqrt{b v}$ is the suction parameter and $B r=\frac{\mu(b x)^{2}}{\kappa\left(T_{w}-T_{\infty}\right)}$ is the Brinkmann number.

The systems of equations (10) and (11) with the boundary condition, equation (12) were solved using the Fourth-Order Runge-Kutta method along with the shooting techniques and results presented in tables and graphically.

\section{Results and Discussion}

\subsection{Numerical Results}

To validate the results, a comparison between the present numerical solution and the works of Makinde and Charles [16] and Arthur and Seini [3] are presented inTable 1. It was observed that the results were consistent with that available in the literature.

Table 1. Comparison of skin friction coefficient, $-f^{\prime \prime}(0)$ for $\lambda$ when $H=0$

\begin{tabular}{llll}
\hline $\boldsymbol{\lambda}$ & $\begin{array}{l}\text { Makinde and } \\
\text { Charles[16] }\end{array}$ & Arthur and Seini [3] & Present Study \\
\hline 0.01 & -0.99802 & -0.99802 & -0.99802 \\
0.02 & -0.99578 & -0.99578 & -0.99578 \\
0.05 & -0.98757 & -0.98757 & -0.98757 \\
0.10 & -0.96938 & -0.96938 & -0.96938 \\
0.20 & -0.91810 & -0.91810 & -0.91810 \\
0.50 & -0.66726 & -0.66726 & -0.66726 \\
2.00 & 2.01750 & 2.01750 & 2.01750 \\
3.00 & 4.72928 & 4.72928 & 4.72928 \\
5.00 & 11.75199 & 11.7520 & 11.7520 \\
\hline
\end{tabular}

The result of varying parameter values on the skin friction coefficient and the rate of heat transfer at the surface is shown in Table 2. It is observed that increasing the velocity ratio parameter decreases the skin friction coefficient and enhances the rate of heat transfer on the surface. This is due to the fact that, in increasing the velocity ratio parameter, an increase in the free stream velocity dominates reducing the drag on the fluid by the surface of the plate and hence enhances the transport of heat.Increasing the magnetic field intensity tends to increase the skin friction due to presence of the Lorenz force induced by the magnetic field in the flow. The Lorenz force retards the fluid flow which results in a decrease of the rate at which heat is transferred. The skin friction and the rate of heat transfer are both increased at the surface as a result of increasing the suction parameter. This is obvious as the porosity in the plate will destruct the usual $x$-directional flow of the fluid on the surface of the plate.

Table 2. Numerical results for varying parameter values

\begin{tabular}{llllllll}
\hline $\boldsymbol{\lambda}$ & $\mathbf{M}$ & $\mathbf{P r}$ & $\boldsymbol{f}_{\boldsymbol{w}}$ & $\mathbf{R a}$ & $\mathbf{B r}$ & $\mathbf{- f ^ { \prime \prime } ( \mathbf { 0 } )}$ & $\boldsymbol{- \theta}^{\prime}(\mathbf{0})$ \\
\hline 0 & 0.1 & 0.71 & 0.1 & 0.1 & 0.1 & 1.10000 & 0.40375 \\
0.5 & 0.1 & 0.71 & 0.1 & 0.1 & 0.1 & 0.76409 & 0.53760 \\
2.0 & 0.1 & 0.71 & 0.1 & 0.1 & 0.1 & -1.97723 & 0.68112 \\
1.0 & 0.5 & 0.71 & 0.1 & 0.1 & 0.1 & 0.37062 & 0.53165 \\
1.0 & 3.0 & 0.71 & 0.1 & 0.1 & 0.1 & 1.50848 & 0.24569 \\
1.0 & 5.0 & 0.71 & 0.1 & 0.1 & 0.1 & 2.06637 & 0.15662 \\
1.0 & 0.1 & 0.72 & 0.1 & 0.1 & 0.1 & 0.08008 & 0.64477 \\
1.0 & 0.1 & 4.00 & 0.1 & 0.1 & 0.1 & 0.08008 & 1.70465 \\
1.0 & 0.1 & 7.20 & 0.1 & 0.1 & 0.1 & 0.08008 & 2.40784 \\
1.0 & 0.1 & 0.71 & 0.5 & 0.1 & 0.1 & 0.09101 & 0.80869 \\
1.0 & 0.1 & 0.71 & 2.0 & 0.1 & 0.1 & 0.13792 & 1.54811 \\
1.0 & 0.1 & 0.71 & 4.0 & 0.1 & 0.1 & 0.20535 & 2.67153 \\
1.0 & 0.1 & 0.71 & 0.1 & 1.0 & 0.1 & 0.08008 & 0.43876 \\
1.0 & 0.1 & 0.71 & 0.1 & 1.5 & 0.1 & 0.08008 & 0.38541 \\
1.0 & 0.1 & 0.71 & 0.1 & 2.0 & 0.1 & 0.08008 & 0.34768 \\
1.0 & 0.1 & 0.71 & 0.1 & 0.1 & 1.0 & 0.08008 & 0.42711 \\
1.0 & 0.1 & 0.71 & 0.1 & 0.1 & 1.5 & 0.08008 & 0.30900 \\
1.0 & 0.1 & 0.71 & 0.1 & 0.1 & 2.0 & 0.08008 & 0.19090 \\
\hline
\end{tabular}


Furthermore, the Prandtl number has no effect on the skin friction coefficient but increases the rate of heat transfer due to the dominance effects of fluid momentum over thermal diffusion. However, increasing both the radiation and the Brinkman numbers keep the skin friction constant whiles the rate of heat transfer reduces due to radiative heating and viscous dissipation.

\subsection{Graphical Results}

\subsubsection{Effects of Parameter Variation on the Velocity Profiles}

The velocity profiles have been plotted to show the behavior of the flow in the boundary layer region. Figures $2-$ 4 present the profiles for varying parameters. Generally, the velocity of the fluid is lowest at the surface of the plate and increases parabolically to the free stream value satisfying the far field boundary condition.

In Figure 2, it is observed that increasing the velocity ratio parameter increases the velocity boundary layer. It is observed that when the velocity ratio is zero, the usual profile at free stream is obtained far away from the surface. The retarding force induced by the increase in the magnetic field intensity reduces the velocity profileas observed in Figure 3. This case is the same for increasing the values of the suction parameter, in Figure 4.

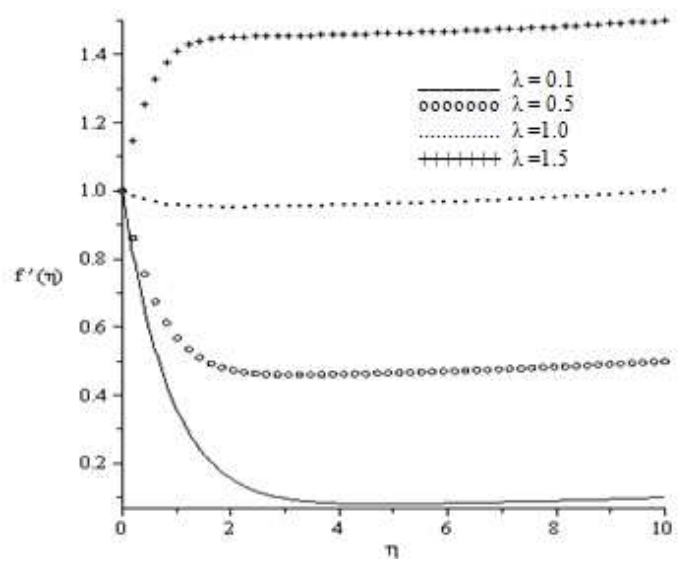

Figure 2. Velocity profile for varying velocity ratio parameter

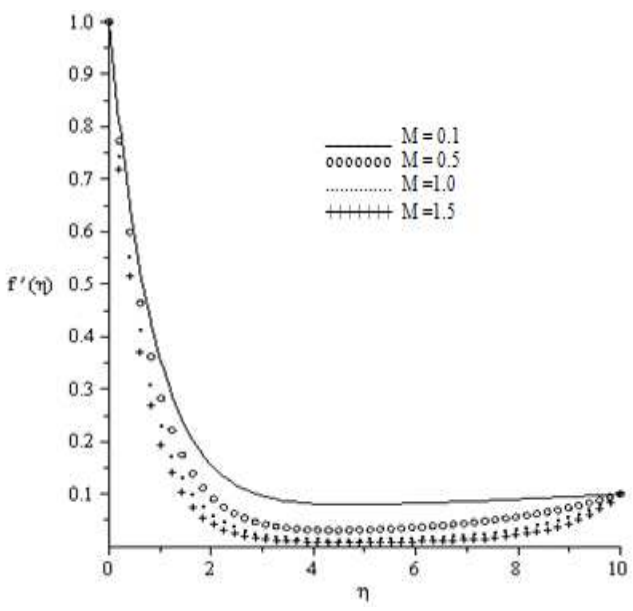

Figure 3. Velocity profiles for varying values of the Magnetic parameter.

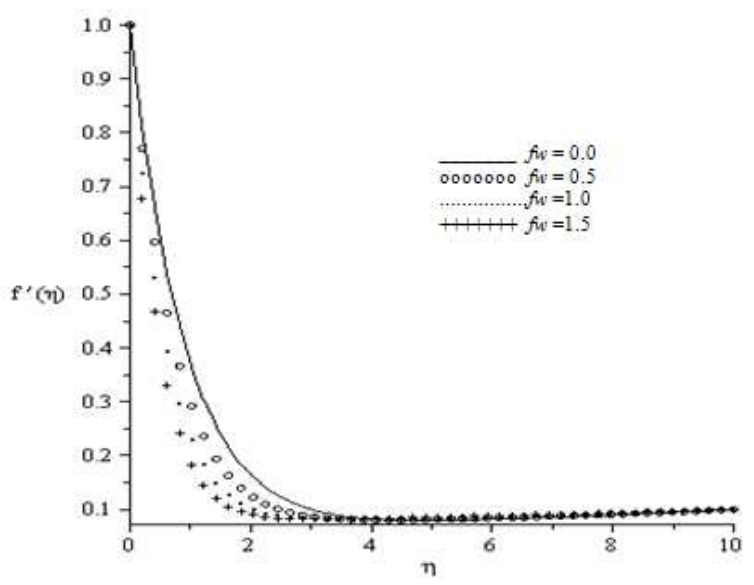

Figure 4. Velocity Profiles for varying suction parameter

\subsubsection{Effects of Parameter Variation of Temperature Profiles}

Figures 5 - 10 present the temperature profiles for various parameter variations. In general, the temperature of the fluid reaches its maximum at the surface of the plate and reduces exponentially to the free stream zero value away from the plate where it attain its minimum, satisfying the boundary condition.

It is observed that increasing the free stream velocity which increases the velocity ratio parameter, reduces the temperature profile at the surface of the plate, figure 7 . Increasing the Prandtl number reduces the thermal boundary layer as a result of increasing momentum of the fluid at the expense of heat diffusion, figure 5.In figure 6 , it is observed that increasing the magnetic parameterincreasesthe thermal boundary layerthicknessfor obvious reasons.

Meanwhile, increasing the radiation parameter increases the temperature profiles due to radiative heating, figure 8 . In figure 9, the suction parameter is observed to reduce the thermal boundary layer thickness. The thermal boundary layer thicknessis observed to increase as a result of increasing the Brinkman number due to viscous dissipation which adds up to heating the fluid, figure 10 . The retarding force of the intensified magnetic field is observed to increase the temperature profiles for obvious reasons.

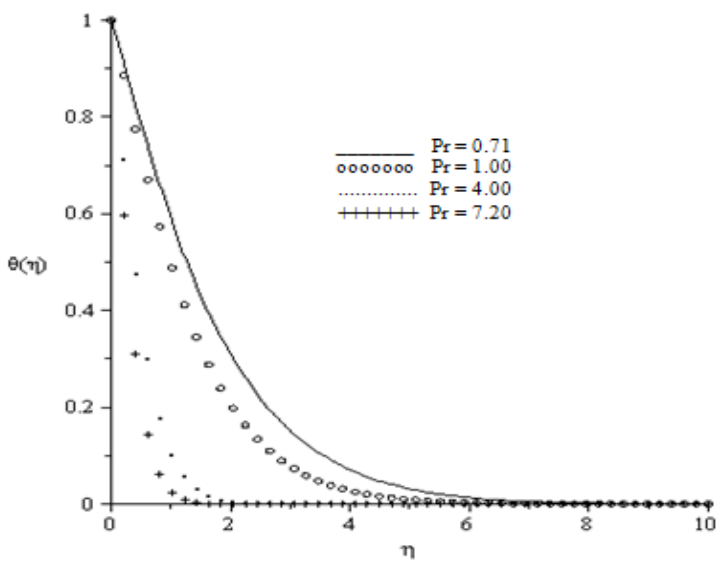

Figure 5. Temperature Profiles for increasing Prandtl number. 


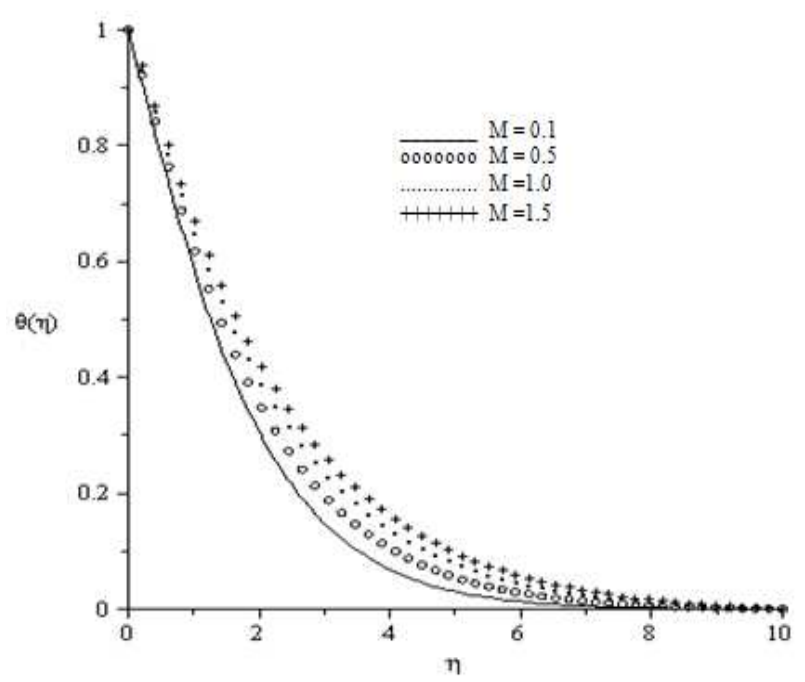

Figure 6. Temperature Profiles for increasing magnetic field parameter.

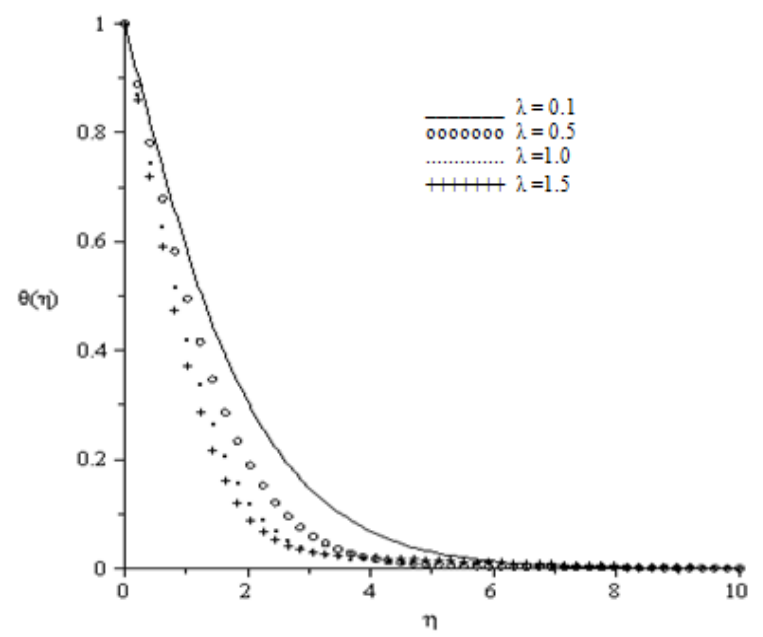

Figure 7. Temperature Profiles for increasing velocity ratio parameter.

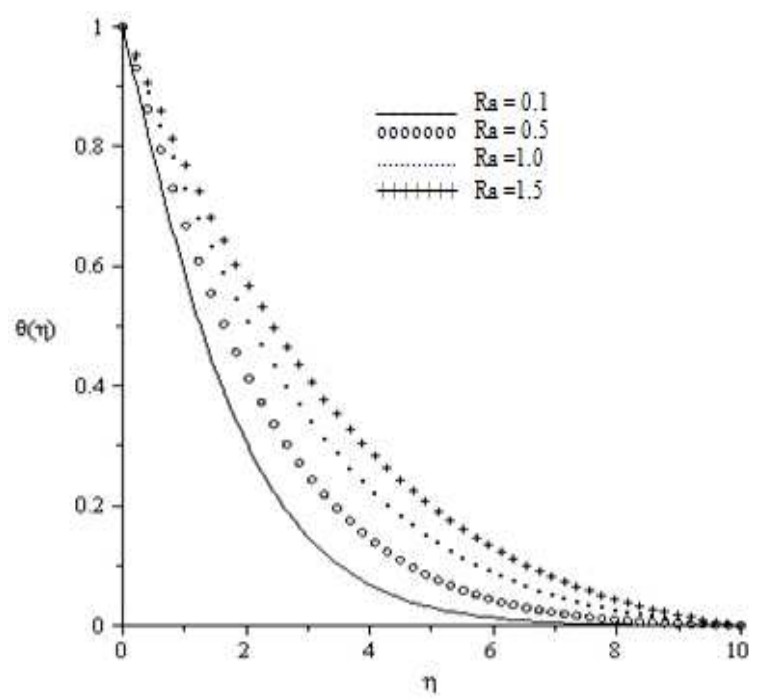

Figure 8. Temperature Profiles for varying Radiation parameter.

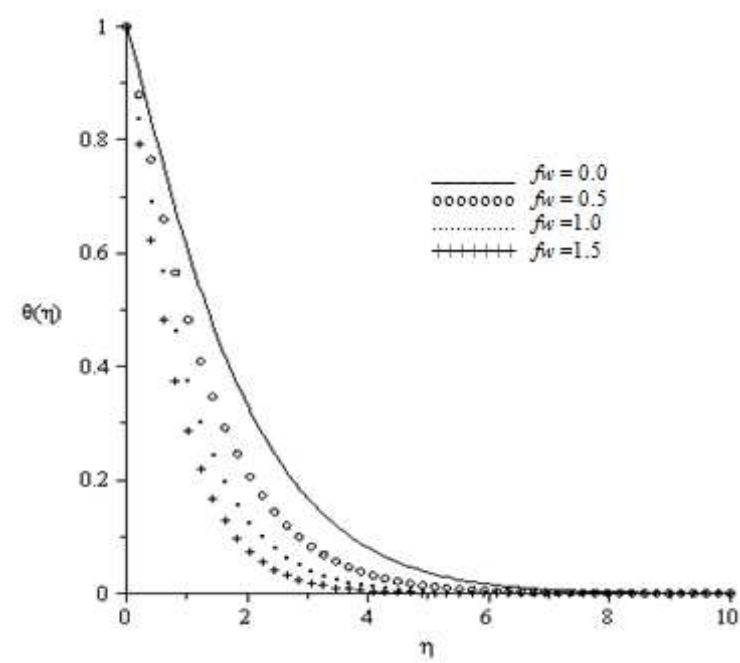

Figure 9. Temperature Profiles for varying Suction Parameter.

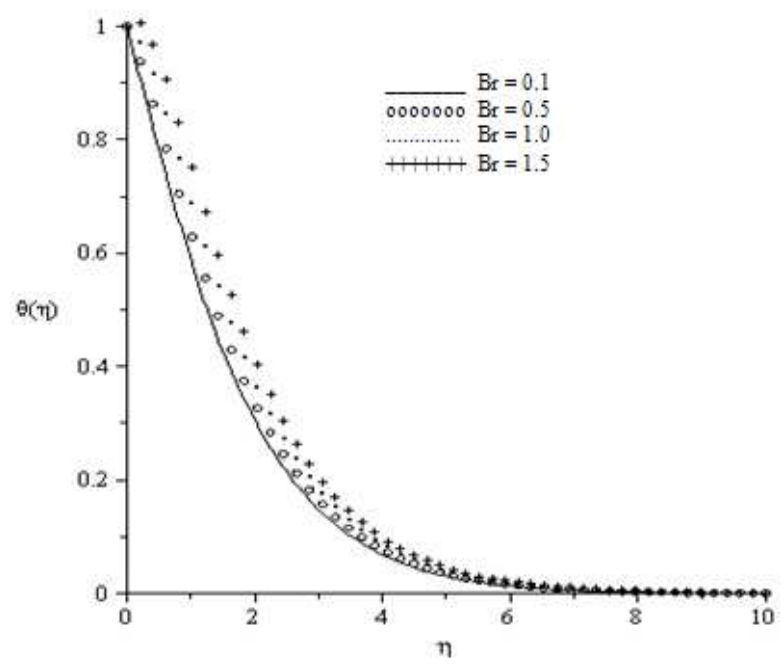

Figure 10. Temperature Profiles for varying Brinkmann Number.

\section{Conclusions}

The MHD thermal stagnation point flow towards a stretching permeable surface has been investigated. Numerical results have been compared to earlier results published in the literature and a perfect agreement was achieved. Among others, our results reveal that the heat transfer of a hydromagnetic fluid flow over a porous stretching sheet subject to radiation and viscous dissipation can be controlled and a final product with desired characteristics achieved.

\section{References}

[1] P. Adrian, "Heat and Mass Transfer by Natural Convection at a Stagnation Point in a Porous Medium Considering Soret and Dufour Effects", Heat Mass Transfer, 46, 2010, pp 831 - 840.

[2] R. Alireza, M. Farzaneh-Gord, S. R Varedi, and D.D. Ganji, "Analytical Solution for Magnetohydrodynamic Stagnation Point Flow and Heat Transfer over a Permeable Stretching Sheet with Chemical Reaction", Journal of Theoretical and Applied Mechanics 51, (3), 2013,pp 675-686, Warsaw. 
[3] E. M Arthur and Y. I. Seini, "MHD Thermal Stagnation Point Flow towards a Stretching Porous Surface",Mathematical Theory and Modeling, 4(5), 2014, pp 163-169.

[4] K. Bhattacharyya and G.C. Layek, "Effects of suction/blowing on steady boundary layer stagnation point flow and heat transfer towards a shrinking sheet with thermal radiation", Int. J. Heat and Mass Transfer, 54, 2011,pp $302-307$.

[5] C.D.S. Devi, H.S. Takhar,and G. Nath, "Unsteady mixed convection flow in stagnation region adjacent to a vertical surface", Heat Mass Trans. 26,1991, pp $71-79$.

[6] P. Dulal,"Heat and mass transfer in stagnation-point flow towards a stretching surface in the presence of buoyancy and thermal radiation", Meccanica, 44, 2009, pp 145-148. DOI 10.1007/s11012-008-9155-1.

[7] C. J. Etwire, Y. I. Seini, and E. M. Arthur,"MHD Boundary Layer Stagnation Point Flow with Radiation and Chemical Reaction towards a Heated Shrinking Porous Surface", International Journal of Physical Science 9(14), 2014, pp 320 328 .

[8] K. Hiemenz, "Die Grenzschicht an einem in dengleichformingenFlussigkeitsstrmeinge-tauchtengradenKrei szylinder”, Dinglers Polytech. J., 326, 1911, pp 321 - 324.

[9] M. A. Hossain and H. S. Takhar, "Radiation effect on mixed convection along a vertical plate with uniform surface temperature", Heat mass transfer 31, 1996, pp $243-248$.

[10] S.Y. Ibrahim and O.D. Makinde, "On MHD boundary layer flow of chemically reacting fluid with heat and mass transfer past a stretching sheet", International Journal of fluid Mechanics, 2(2), 2010, pp $123-132$.

[11] S.Y. Ibrahim and O.D. Makinde,"Radiation Effect on Chemically Reacting MHD Boundary Layer Flow of Heat and Mass Transfer past a Porous Vertical Flat Plate", International Journal of the Physical Sciences, 6(6), 2011, pp 1508 - 1516.

[12] A. Ishak, K. Jafar,R. Nazar, and, I. Pop, “ MHD stagnation point flow towards a stretching sheet", Physica A, 388, 2009, pp $3377-3383$.

[13] S.A. Kechil and I. Hashim, (), Series solution of flow over nonlinearly stretching sheet with chemical reaction and magnetic field, Physics Letters B, 372, 2008, pp 2258 - 2263.

[14] G. C. Layek, S., Mukhopadhyay, and S. A. Samad, "Heat and mass transfer analysis for boundary layer stagnation point flow towards a heated porous stretching sheet with heat absorption/generation and suction/blowing," International Communications in Heat and Mass Transfer, 34 (3), 2007, pp 347-356.

[15] Y. Y. Lok, N. Amin, D. Campean, and I. Pop,"Steady mixed convection flow of a micropolar fluid near the stagnation point on a vertical surface, Int. J. Numer. Meth. Heat Fluid Flow", 15, 2005, pp 654-670.

[16] O.D. Makinde and W.M. Charles, "Computational dynamics of hydromagnetic stagnation flow towards a stretching sheet", Appl. Comput. Math., 9(2), 2010, pp 243 - 251.

[17] S. R. Pop, T. Grosan, and I. Pop, "Radiation effects on the flow near the stagnation point of a stretching sheet", Tech. Mech., Band 25. Heft 2, 2004, pp $100-106$.

[18] N. Ramachandran, T. S.Chen, and B. F. Armaly,"Mixed convection in stagnation flows adjacent to a vertical surface", ASME J. Heat Transfer, 110, 1988, pp $373-377$.

[19] B.C. Sakiadis,"Boundary layer behavior on continuous solid surfaces: I boundary layer equations for two dimensional and axisymmetric flow", American Institute of Chemical Engineers, 61, 1971, pp $26-34$.

[20] Y.I. Seini,"Flow over unsteady stretching surface with chemical reaction and non-uniform heat source", Journal of Engineering and Manufacturing Technology, JEMT 1, 2013,pp $24-35$.

[21] Y.I. Seini and O.D. Makinde, "MHD boundary layer flow due to exponential stretching surface with radiation and chemical reaction", Mathematical Problems in Engineering, 2013. http://dx.doi.org/10.1155/2013/163614.

[22] Y.I. Seini and O.D. Makinde, "Boundary Layer Flow near Stagnation-Points on a Vertical Surface with Slip in the Presence of Transverse Magnetic Field", International Journal of Numerical Methods and Fluid Flow, Vol. 24(3), 2014, pp $643-653$.

[23] J. Zhu, L.C. Zheng, and X.X. Zhang, "The influence of thermal radiation on MHD stagnation point flow past a stretching sheet with heat generation”, Acta. Mech. Sin., 27(4),2011, pp $502-$ 509.

[24] T. Fang, J. Zhang, and S. Yao,"Slip MHD viscous flow over a stretching sheet - an exact solution", Communications in Nonlinear Science and Numerical Simulation, 14, 2009, pp 3731-3737 